\title{
The Role of MDM2 In Neuroendocrine Tumors: A Novel Therapeutic Target
}

\author{
Reddy Priyanka and Amr Mohamed* \\ Department of Hematology and Oncology, University Hospitals, Seidman Cancer Center Case Comprehensive Cancer Center, Case Western \\ Reserve University Cleveland, OH, USA
}

*Corresponding author: Amr Mohamed, Assistant Professor, GI Medical Oncology, Seidman Cancer Center, Case Comprehensive Cancer Center, Case Western Reserve University Cleveland, OH, USA.

\section{Introduction}

Neuroendocrine tumors (NETs) are neoplasms derived from neuroendocrine cells which secrete hormones that cause distinct clinical syndromes. They are considered rare tumors, but annual incidence has increased significantly with recent SEER analysis showing a 6.4-fold increased incidence from 1973 (1.09 per $100,000)$ to 2012 (6.98 per 100,000) [1]. They are highly diverse neoplasms that can be divided into several subgroups based on embryonic derivation, histology, functionality, and origin of primary tumor. One of the most important classifications, based on histologic differentiation and grade, divides NETs into well-differentiated and poorly differentiated neoplasms [2]. Well-differentiated NETs are indolent tumors with a slow progression profile, while poorly differentiated neuroendocrine carcinomas (NECs) are aggressive tumors with worse outcomes [3]. Well-differentiated and poorly differentiated tumors have vastly different responses to treatment and thus require different clinical approaches [2]. While there have been therapeutic advances in NETs during the last decade, treatment options are still limited, especially in those with higher grade tumors. The multiple types of NETs and the differences in their clinical behavior demonstrates the need for further research and the development of more targeted therapies. Given this, a more detailed understanding of the molecular pathogenesis and the development of novel therapeutics is needed.

While most poorly differentiated NECs harbor alterations in tumor protein p53 (TP53) and retinoblastoma (RB1) genes, there is limited data about their expression/alteration in other NET subgroups and how these features may contribute to the separation of well from poorly differentiated NETs with a proliferation index above 20\% [4]. There is also little known about the relationship between the transcription factor $\mathrm{p} 53$ pathway and the E3 ubiquitinprotein ligase mouse double minute 2 homologue (MDM2; also called HDM2 for its human homologue). Given the paucity of genetic information regarding MDM2-TP53 pathway in NETs, we will summarize the role of MDM2 overexpression and its relation to TP53 as a potential targeted therapy in preclinical models and early phase clinical trials in these neoplasms.

\section{TP53 and MDM2}

Transcription factor p53 has been called "the guardian of the genome" because it plays a critical role in regulating genomic stability and in suppression of tumor development. It is a haploinsufficiency gene in both mice and humans that is located on chromosome 17 p13.1 and is essential in cell division as it regulates cell cycle phases [5]. TP53 itself is closely regulated by a number of positive and negative regulators and any small change in activity can deregulate the cell cycle, cause uncontrolled cell division, and predispose to tumorigenesis [5]. As more than 50\% of all tumor types have an impaired p53 signaling pathway, researchers have successfully identified many downstream transcription targets for p53 [6]. One of these targets is the E3 ubiquitin-protein ligase mouse double minute 2 homologue (MDM2). Since discovery by Dona George in 1991, many in vitro and in vivo studies have described the tangled relationship between MDM2 and p53 [7]. The human MDM2 gene is located on chromosome 12 (q14.3-q15), a region with many genes thought to be involved in control of cell growth [8]. Reports indicate that MDM2 binds with wild type p53, targeting it for proteolytic degradation and thus inhibiting p53mediated gene expression $[9,10]$. It has been found that by adding exogenous MDM2, p53-mediated G1 phase cell cycle arrest and induction of apoptosis can be suppressed [8]. Additionally, p53 has been found to transactivate MDM2 expression suggesting that the MDM2 and TP53 complex may autoregulate MDM2 itself as well as TP53 function $[8,10]$. 
MDM2 also plays a critical role in carcinogenesis in a p53independent manner [8]. In addition to a p53 binding domain, MDM2 contains other domains that suggest that it can bind to DNA and affect transcription. It has been reported that MDM2 binds to retinoblastoma (RB) tumor suppressor protein as well as other transcription factors [8]. Preclinical studies showed the formation of these complexes in vitro and in cultured cells deficient of p53, therefore suggesting that MDM2 might play a p53-independent role in promoting cell cycle progression from G1 to $S$ phase [8].

\section{Role of P53 and MDM2 in different groups of neu- roendocrine tumors}

While p53 and MDM2 have been studied in multiple tumor types, the data regarding their role in neuroendocrine tumors is limited [11]. There are several differences in the mutational frequencies between high grade well-differentiated NETs and poorly differentiated NECs. TP53 mutations and alterations in expression of the RB1 gene seem to be mainly in poorly differentiated NECs. Previous studies have analyzed the difference in p53 and its regulatory molecules in well-differentiated versus poorly differentiated neuroendocrine tumors. In one study of 63 patients with NETs, mutated TP53 was the most prevalent mutation (57\%) in poorly differentiated NECs and was rarely detected in those with well-differentiated NETs (4). Thus, this study demonstrated the importance of the p53 pathway in poorly differentiated NECs and the importance in understanding the genetic differences between poorly differentiated and well-differentiated NETs.

While TP53 mutations are rare in well-differentiated NETs, the underlying molecular mechanisms regarding TP53 and its key regulators in well-differentiated NETs are largely unknown and there are suggestions that there still may be a role of the TP53 pathway in contributing to tumorigenesis via regulatory molecules. A study of the genetic alterations in well-differentiated pancreatic NETs confirmed that TP53 mutations were rare in welldifferentiated pNETs $(<3 \%)$ [5]. However, the results also detected that about $70 \%$ of pNETs had an altered p53 pathway through aberrant activation of its negative regulators including MDM2 (22\%), MDM4 (30\%), and WIP1 (51\%). These results clearly demonstrated that although most patients with well-differentiated pancreatic NETs have wild TP53, they do exhibit negative regulation of TP53 function which could be an important mechanism for the pNETs progression. Therefore, reactivation of TP53 could be a potential therapeutic strategy for these patients.

\section{Targeting MDM2}

Given the previous data highlighting the potential role of MDM2TP53 pathway in NETs' tumorigenesis, several preclinical studies targeted MDM2 in NETs in vitro. In one study, MDM2 inhibitor, NVP-CGM097, was evaluated in NETs from different origins include mutated p53 pancreas (BON1), and lung (NCI-H727), and wild p53 midgut (GOT1) cell lines [12]. Each tumor cell line was incubated with NVP-CGM097 at different concentrations that ranged from 4-2,500 Nm. The study showed that wild type p53 GOT1 tumor cells of the midgut were sensitive and more responsive to NVP-CGM097 at higher concentrations. Co-incubation with other chemotherapies and targeted agents including 5-fluorouracil, temozolomide, or everolimus revealed increased anti-proliferative tumor effects in GOT1 cells [12]. The mutated p53 NCI-H727 and BON1 were resistant to NVP-CGM097. While this study encourages further workup of MDM2 inhibition for wild type p53 NETs, it also reveals that mutated p53 NETs are resistant to MDM2 inhibitors.

Another study analyzed nutlin-3a, a molecule that inhibits the MDM2-p53 pathway [13]. The study demonstrated that MDM2 was overexpressed in intestinal NETs and was higher in metastatic than in localized disease. The results confirmed the previous findings of wild p53 small intestinal NET cell lines exhibiting cell cycle arrest and apoptosis through nutlins reactivating a p53 antiproliferative response [13]. The antitumor effects of nutlin-3a were also confirmed in vivo in a xenograft mouse model. Additionally, the data suggested that MDM2 also influences the expression of oncogene FOXM1 in a p53-independent manner. Overall, these results demonstrated MDM2 overexpression in NETs and that targeting MDM2 overexpression could become a broad and clinically viable therapeutic strategy for NETs with wild-type p53.

\section{Conclusion}

The underlying molecular mechanisms driving NETs resistance are largely unknown and remain a significant scientific barrier. Although NETs show indolent clinical behavior, these tumors do in fact exhibit significant genomic instability. Therefore, discovering new therapeutic targets based on the biology of this unmet clinical need is highly significant. There are several preclinical studies that have analyzed MDM2 overexpression in relation to TP53 gene status and its impact on tumorigenesis of NETs. Based on preclinical data, MDM2 inhibitors are considered promising new therapeutic targets. Despite this, there is still a significant need for a deeper understanding of the underlying molecular genetics of the MDM2/MDMX-Tp53 axis in different NETs subgroups due to the complexity and heterogeneity of the disease. Future research should focus on differentiating MDM2 expression in well versus poorly differentiated tumors as well as according to origin of the primary tumor. Given the differences in treatment response and outcome between pancreatic and midgut NETs, MDM2 and TP53 needs to be analyzed in each subgroup separately. Additionally, future research should also provide a better assessment of the biological effects and toxicities of targeting MDM2 in NET patients in early phase clinical trials.

\section{References}

1. Dasari A, Shen C, Halperin D (2017) Trends in the Incidence, Prevalence, and Survival Outcomes in Patients with Neuroendocrine Tumors in the United States. JAMA Oncol 3(10): 1335-1342. 
2. Rindi G, Klimstra DS, Abedi Ardekani B (2018) A common classification framework for neuroendocrine neoplasms: an International Agency for Research on Cancer (IARC) and World Health Organization (WHO) expert consensus proposal. Mod Pathol 31:1770-1786.

3. Sigel CS, Krauss Silva VW, Reid MD, Chhieng D, Basturk O, et al. (2018) Well differentiated grade 3 pancreatic neuroendocrine tumors compared with related neoplasms: A morphologic study. Cancer Cytopathology 126: 326-335.

4. Vijayvergia N, Boland P, Handorf E et al. (2016) Molecular profiling of neuroendocrine malignancies to identify prognostic and therapeutic markers: a Fox Chase Cancer Center Pilot Study. Br J Cancer 115: 564570.

5. Taneja P, Zhu S, Maglic D, Fry EA, Kendig RD, et al. (2011) Transgenic and knockout mice models to reveal the functions of tumor suppressor genes. Clin Med Insights Oncol 5: 235-257.

6. Hu W, Feng Z, Modica I, Klimstra DS, Song L, Allen PJ, et al. (2010) Gene Amplifications in Well-Differentiated Pancreatic Neuroendocrine Tumors Inactivate the p53 Pathway. Genes Cancer 1(4): 360-368.

7. Fakharzadeh SS, Trusko SP, George DL (1991) Tumorigenic potential associated with enhanced expression of a gene that is amplified in a mouse tumor cell line. EMBO J 10(6): 1565-1569.
8. Jones SN, Hancock AR, Vogel H, Donehower LA, Bradley A (1998) Overexpression of $\mathrm{Mdm} 2$ in mice reveals a p53-independent role for Mdm2 in tumorigenesis. Proc Natl Acad Sci USA 95(26): 15608-15612.

9. Wade M, Li YC, Wahl GM (2013) MDM2, MDMX and p53 in oncogenesis and cancer therapy. Nat Rev Cancer 13(2): 83-96.

10. Shangary S, Wang S (2008) Targeting the MDM2-p53 interaction for cancer therapy. Clin Cancer Res 14(17): 5318-5324.

11. Rayburn E, Zhang R, He J, Wang H (2005) MDM2 and human malignancies: expression, clinical pathology, prognostic markers, and implications for chemotherapy. Curr Cancer Drug Targets 5(1): 27-41.

12. Reuther C, Heinzle V, Nolting S, Herterich S, Hahner S, et al. (2018) The HDM2 (MDM2) Inhibitor NVP-CGM097 Inhibits Tumor cell Proliferation and Shows Additive Effects with 5-Fluorouracil on the p53 - p21 - Rb - E2F1 Cascade in the p53wildtype Neuroendocrine Tumor Cell Line GOT1. Neuroendocrinology 106(1):1-19.

13. Briest F, Grass I, Sedding D (2018) Mechanisms of Targeting the MDM2p53-FOXM1 Axis in Well-Differentiated Intestinal Neuroendocrine Tumors. Neuroendocrinology 107(1): 1-23.

\section{(c) (1) \\ This work is licensed under Creative Commons Attribution 4.0 License}

To Submit Your Article Click Here: Submit Article

DOI: $10.32474 /$ RRHOAJ.2020.05.000217

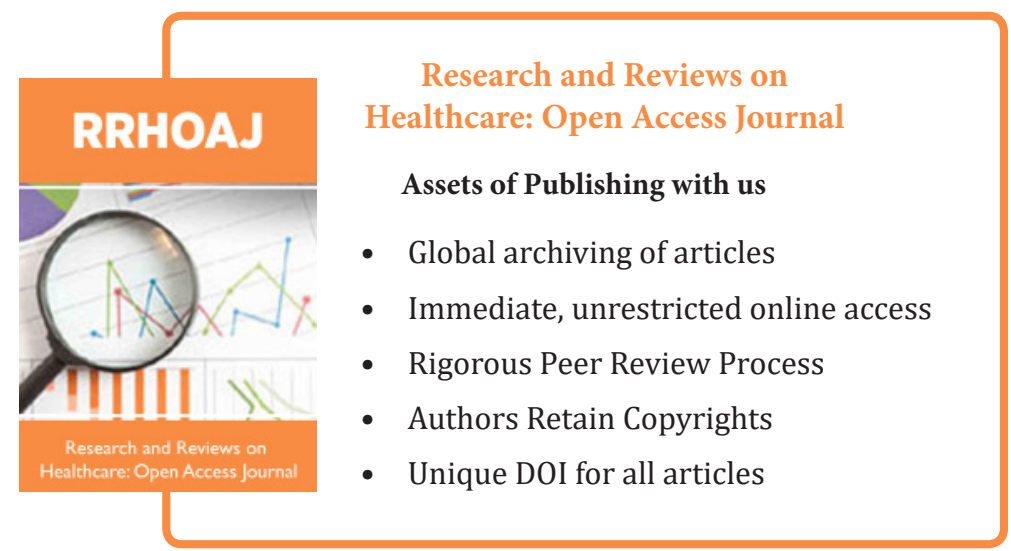

